Acta Crystallographica Section E

Structure Reports

Online

ISSN 1600-5368

\section{Thomas C. Lewis and \\ Derek A. Tocher*}

Department of Chemistry, University College London, 20 Gordon Street, London, WC1H OAJ, England

Correspondence e-mail: d.a.tocher@ucl.ac.uk

\section{Key indicators}

Single-crystal X-ray study

$T=150 \mathrm{~K}$

Mean $\sigma(\mathrm{C}-\mathrm{C})=0.001 \AA$

Disorder in main residue

$R$ factor $=0.028$

$w R$ factor $=0.078$

Data-to-parameter ratio $=10.4$

For details of how these key indicators were automatically derived from the article, see

http://journals.iucr.org/e.

\title{
Redetermination of 5,5-dihydroxybarbituric acid trihydrate (alloxan tetrahydrate)
}

The low temperature redetermination of 5,5-dihydroxybarbituric acid trihydrate, $\mathrm{C}_{4} \mathrm{H}_{4} \mathrm{~N}_{2} \mathrm{O}_{5} \cdot 3 \mathrm{H}_{2} \mathrm{O}$, (historically misnamed alloxan tetrahydrate) is reported here. The organic molecule has crystallographic mirror symmetry, as does one of the water molecules.

\section{Comment}

The crystal structure of 5,5-dihydroxybarbituric acid trihydrate, (I) (originally misnamed alloxan tetrahydrate) was determined by Mootz \& Jeffrey (1965). In that original roomtemperature study, only three of the six $\mathrm{H}$ atoms in the asymmetric unit could be located by means of difference Fourier syntheses, and the structure refined to a final $R$ value of 0.097 . We have redetermined this crystal structure at $150 \mathrm{~K}$, with a final $R$ value of 0.028 , to gain more accurate data for our theoretical modelling studies. The low-temperature redetermination located all the $\mathrm{H}$ atoms, which were refined isotropically. The precision of the unit-cell dimensions was improved by an order of magnitude. The unit-cell volume decreased by $c a$. $27 \AA^{3}$, consistent with the determination at low temperature. In general, the molecular geometric parameters are not significantly different, the exception being the C6-O6 bond length, which is shorter in the lowtemperature structure, while $\mathrm{C} 2-\mathrm{O} 2$ is actually longer in the low-temperature structure, both by $c a$. $0.1 \AA$.

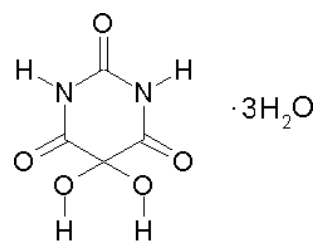

(I)

Compound (I) crystallizes in the monoclinic space group $C 2 / m$, with the organic molecule on a mirror plane plus one water molecule in a general position and a second on a mirror plane (Fig. 1). The crystallographic plane is normal to the pyrimidine ring, passing through atoms $\mathrm{O} 2, \mathrm{C} 2, \mathrm{C} 5, \mathrm{O} 7$ and O8. The hydroxyl $\mathrm{H}$ atoms attached to the ring are disordered across the mirror plane. The water molecule $\mathrm{O} 2 W$, in a general position, is disordered with one of the $\mathrm{H}$ atoms refined over two positions. The third water of solvation lies on the mirror plane at $(0, y, 1)$, with the mirror bisecting the $\mathrm{H}-\mathrm{O}-\mathrm{H}$ angle.

The $\mathrm{C}-\mathrm{N}$ bond lengths in the ring range from 1.3666 (11) to 1.3752 (9) $\AA$, and the C5 - C6 bond length is 1.5272 (10) $\AA$. The packing (Fig. 2) consists of centrosymmetric dimers
Received 25 August 2004 Accepted 6 September 2004 Online 18 September 2004 

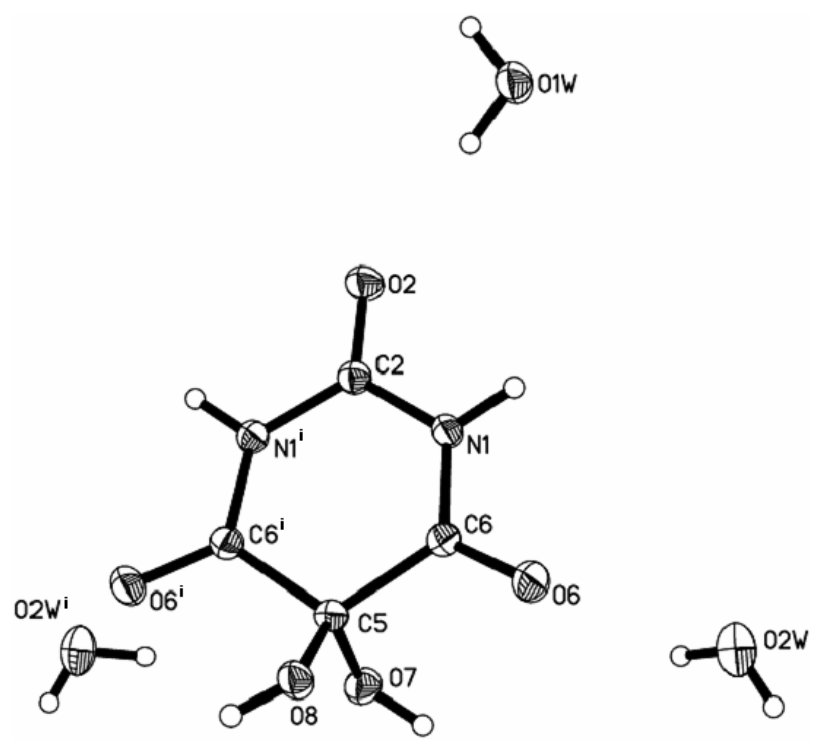

Figure 1

View of the 5,5-dihydroxybarbituric acid molecule and three water molecules (twice the asymmetric unit), showing the atom labelling scheme. Displacement ellipsoids are drawn at the $50 \%$ probability level. [Symmetry code: (i) $x,-y, z$.]

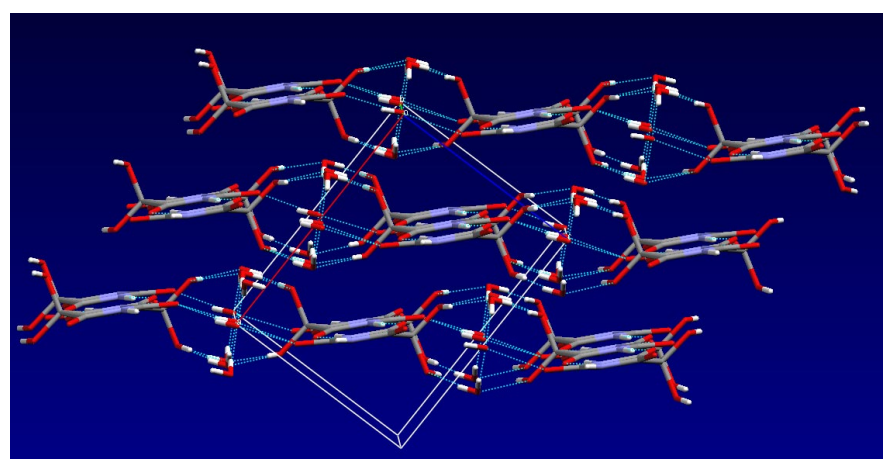

Figure 2

The crystal packing of 5,5-dihydroxybarbituric acid trihydrate, showing the $\mathrm{N}-\mathrm{H} \cdots \mathrm{O}$ and $\mathrm{O}-\mathrm{H} \cdots \mathrm{O}$ hydrogen-bonding interactions as dashed lines.

hydrogen-bonded to form a chain, with the water molecules lying between these chains, forming a buckled sheet structure. The water molecules in general positions form $\mathrm{O}-\mathrm{H} \cdots \mathrm{O}$ bonds to the organic hydroxyl groups, whilst the water molecules on the mirror plane bond to the unique hydrogen-bond carbonyl acceptor on the organic molecules (Fig. 3). The water molecules on the mirror planes and in general positions also hydrogen-bond to each other in the sheet. The $D \cdots A$ distance within the bonded chains of molecules is 2.8580 (6) $\AA$, whilst the $\mathrm{O}-\mathrm{H} \cdots \mathrm{O}$ hydrogen bonds range from 2.7217 (11) to 2.9343 (9) $\AA$. All potential donors and acceptors are used in the hydrogen bonding.

\section{Experimental}

To complement the results from an experimental polymorph search on alloxan, 5,5-dihydroxybarbituric acid trihydrate was obtained from Aldrich as colourless plate-like crystals of alloxan tetrahydrate

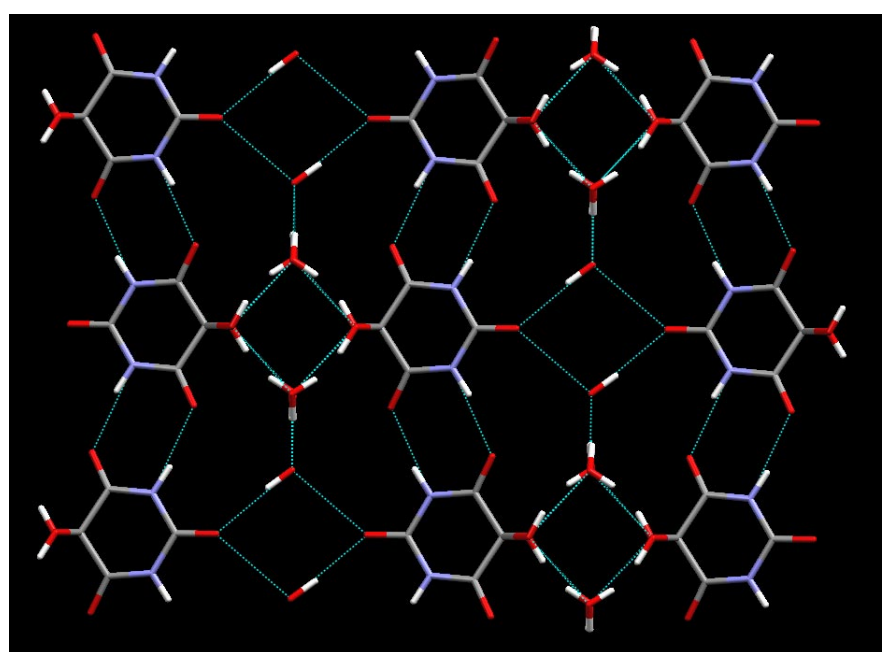

Figure 3

The hydrogen-bonded sheet structure in 5,5-dihydroxybarbituric acid trihydrate, showing the $\mathrm{N}-\mathrm{H} \cdots \mathrm{O}$ and $\mathrm{O}-\mathrm{H} \cdots \mathrm{O}$ hydrogen-bonding interactions as dashed lines.

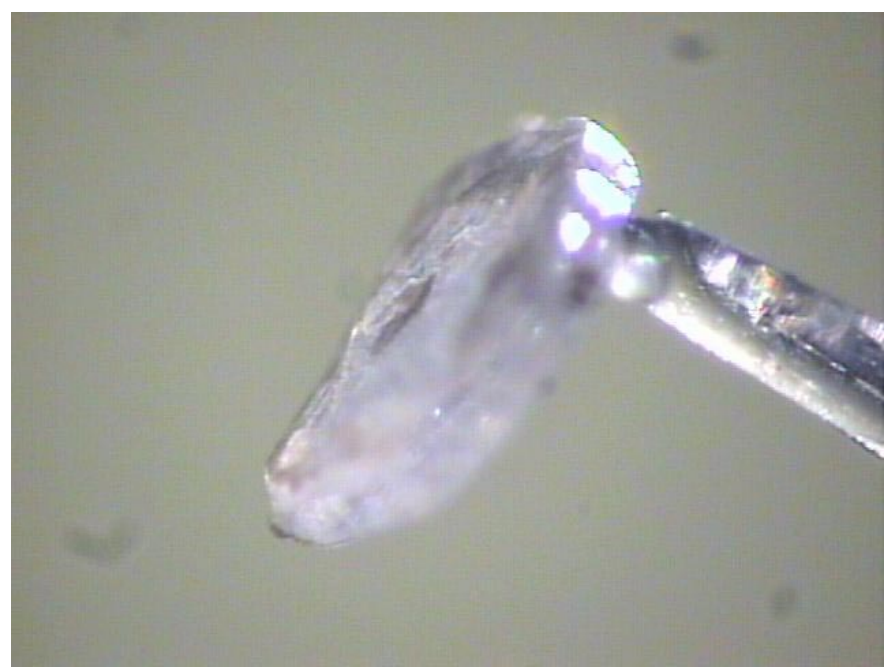

Figure 4

The crystal morphology of 5,5-dihydroxybarbituric acid trihydrate.

(Fig. 4). These crystals were very sensitive and decompose rapidly in air.

\section{Crystal data}

$\mathrm{C}_{4} \mathrm{H}_{4} \mathrm{~N}_{2} \mathrm{O}_{5} \cdot 3 \mathrm{H}_{2} \mathrm{O}$

$M_{r}=214.14$

Monoclinic, $C 2 / m$

$a=9.4614(8) \AA$

$b=12.2095(10) \AA$

$c=7.2973(6) \AA$

$\beta=91.4650(10)^{\circ}$

$V=842.70(12) \AA^{3}$

$Z=4$

$D_{x}=1.688 \mathrm{Mg} \mathrm{m}^{-3}$

Mo $K \alpha$ radiation

Cell parameters from 3125

reflections

$\theta=2.7-28.2^{\circ}$

$\mu=0.17 \mathrm{~mm}^{-1}$

$T=150(2) \mathrm{K}$

Plate, colourless

$0.72 \times 0.62 \times 0.11 \mathrm{~mm}$

\section{Data collection}

Bruker SMART APEX

diffractometer

$\omega$ scans

Absorption correction: multi-scan

(SADABS; Sheldrick, 1996)

$T_{\min }=0.889, T_{\max }=0.982$

3701 measured reflections
1036 independent reflections 1019 reflections with $I>2 \sigma(I)$

$R_{\text {int }}=0.015$

$\theta_{\max }=28.2^{\circ}$

$h=-12 \rightarrow 12$

$k=-15 \rightarrow 15$

$l=-9 \rightarrow 9$ 


\section{organic papers}

\section{Refinement}

Refinement on $F^{2}$ $R\left[F^{2}>2 \sigma\left(F^{2}\right)\right]=0.028$

$w R\left(F^{2}\right)=0.078$

$S=1.12$

1036 reflections

100 parameters

All H-atom parameters refined

$$
\begin{aligned}
& w=1 /\left[\sigma^{2}\left(F_{o}{ }^{2}\right)+(0.0445 P)^{2}\right. \\
& +0.3397 P] \\
& \text { where } P=\left(F_{o}^{2}+2 F_{c}^{2}\right) / 3 \\
& (\Delta / \sigma)_{\max }<0.001 \\
& \Delta \rho_{\max }=0.41{\mathrm{e} \AA^{-3}}^{-3} \\
& \Delta \rho_{\min }=-0.24{\mathrm{e} \AA^{-3}}^{-3}
\end{aligned}
$$

Table 1

Hydrogen-bonding geometry $\left(\AA \stackrel{\circ}{ }^{\circ}\right)$.

\begin{tabular}{lllll}
\hline$D-\mathrm{H} \cdots A$ & $D-\mathrm{H}$ & $\mathrm{H} \cdots A$ & $D \cdots A$ & $D-\mathrm{H} \cdots A$ \\
\hline $\mathrm{N} 1-\mathrm{H} 1 \cdots \mathrm{O} 6^{\mathrm{i}}$ & $0.901(14)$ & $1.970(14)$ & $2.8580(10)$ & $168.1(13)$ \\
$\mathrm{O} 7-\mathrm{H} 7 \cdots \mathrm{O} 2 W^{\mathrm{ii}}$ & $0.83(3)$ & $1.92(3)$ & $2.7217(11)$ & $163(3)$ \\
$\mathrm{O} 8-\mathrm{H} 8 \cdots \mathrm{O} 2 W^{\mathrm{iii}}$ & $0.78(3)$ & $1.98(3)$ & $2.7250(10)$ & $160(3)$ \\
$\mathrm{O} 1 W-\mathrm{H} 9 \cdots \mathrm{O} 2$ & $0.868(18)$ & $2.075(19)$ & $2.9343(9)$ & $170.6(16)$ \\
$\mathrm{O} 2 W-\mathrm{H} 10 \cdots \mathrm{O} 1 W^{\mathrm{iv}}$ & $0.85(2)$ & $1.96(2)$ & $2.8009(11)$ & $171.3(15)$ \\
$\mathrm{O} 2 W-\mathrm{H} 11 A \cdots 7^{\mathrm{ii}}$ & $0.77(4)$ & $1.99(4)$ & $2.7217(11)$ & $160(3)$ \\
$\mathrm{O} 2 W-\mathrm{H} 11 B \cdots 8^{\mathrm{v}}$ & $0.82(3)$ & $1.96(3)$ & $2.7250(10)$ & $154(3)$
\end{tabular}

Symmetry codes: (i) $\frac{1}{2}-x, \frac{1}{2}-y, 1-z$; (ii) $\frac{1}{2}-x, \frac{1}{2}-y,-z$; (iii) $\frac{1}{2}+x, y-\frac{1}{2}, z$; (iv) $x, y, z-1 ;$ (v) $x-\frac{1}{2}, \frac{1}{2}+y, z$.

The non-H atoms were refined freely with anisotropic displacement parameters, with the $\mathrm{H}$ atoms refined independently with an isotropic model.
Data collection: SMART (Bruker, 2000); cell refinement: SAINT (Bruker, 2000); data reduction: SAINT; $\operatorname{program}(\mathrm{s})$ used to solve structure: SHELXS97 (Sheldrick, 1990); program(s) used to refine structure: SHELXL97 (Sheldrick, 1997); molecular graphics: MERCURY (Bruno et al., 2002) and SHELXTL (Bruker, 2000); software used to prepare material for publication: SHELXL97.

This research was supported by the EPSRC in funding a studentship for TCL. The authors acknowledge the Research Councils UK Basic Technology Programme for supporting 'Control and Prediction of the Organic Solid State'. For more information on this work, please visit http://www.chem. ucl.ac.uk/basictechorg/.

\section{References}

Bruker (2000). SMART, SAINT and SHELXTL. Bruker AXS Inc., Madison, Wisconsin, USA.

Bruno, I. J., Cole, J. C., Edgington, P. R., Kessler, M. K., Macrae, C. F., McCabe, P., Pearson, J. \& Taylor, R. (2002). Acta Cryst. B58, 389-397.

Mootz, D., Jeffrey, G. A. (1965). Acta Cryst. 19, 717-725.

Sheldrick, G. M. (1990). Acta Cryst. A46, 467-473.

Sheldrick, G. M. (1996). SADABS. University of Göttingen, Germany.

Sheldrick, G. M. (1997). SHELXL97. University of Göttingen, Germany. 


\section{supporting information}

Acta Cryst. (2004). E60, o1748-o1750 [https://doi.org/10.1107/S1600536804022019]

\section{Redetermination of 5,5-dihydroxybarbituric acid trihydrate (alloxan}

\section{tetrahydrate)}

\section{Thomas C. Lewis and Derek A. Tocher}

5,5-dihydroxybarbituric acid trihydrate

Crystal data

$\mathrm{C}_{4} \mathrm{H}_{4} \mathrm{~N}_{2} \mathrm{O}_{5} \cdot 3 \mathrm{H}_{2} \mathrm{O}$

$M_{r}=214.14$

Monoclinic, $C 2 / m$

$a=9.4614(8) \AA$

$b=12.2095(10) \AA$

$c=7.2973(6) \AA$

$\beta=91.465(1)^{\circ}$

$V=842.70(12) \AA^{3}$

$Z=4$

\section{Data collection}

Bruker SMART APEX

diffractometer

Radiation source: fine-focus sealed tube

Graphite monochromator

$\omega$ rotation with narrow frames scans

Absorption correction: multi-scan

(SADABS; Sheldrick, 1996)

$T_{\min }=0.889, T_{\max }=0.982$

\section{Refinement}

Refinement on $F^{2}$

Least-squares matrix: full

$R\left[F^{2}>2 \sigma\left(F^{2}\right)\right]=0.028$

$w R\left(F^{2}\right)=0.078$

$S=1.12$

1036 reflections

100 parameters

0 restraints

Primary atom site location: structure-invariant direct methods
$F(000)=448$

$D_{\mathrm{x}}=1.688 \mathrm{Mg} \mathrm{m}^{-3}$

Mo $K \alpha$ radiation, $\lambda=0.71073 \AA$

Cell parameters from 3125 reflections

$\theta=2.7-28.2^{\circ}$

$\mu=0.17 \mathrm{~mm}^{-1}$

$T=150 \mathrm{~K}$

Plate, colourless

$0.72 \times 0.62 \times 0.11 \mathrm{~mm}$

3701 measured reflections

1036 independent reflections

1019 reflections with $I>2 \sigma(I)$

$R_{\text {int }}=0.015$

$\theta_{\text {max }}=28.2^{\circ}, \theta_{\min }=2.7^{\circ}$

$h=-12 \rightarrow 12$

$k=-15 \rightarrow 15$

$l=-9 \rightarrow 9$

Secondary atom site location: difference Fourier map

Hydrogen site location: inferred from neighbouring sites

All $\mathrm{H}$-atom parameters refined

$w=1 /\left[\sigma^{2}\left(F_{\mathrm{o}}^{2}\right)+(0.0445 P)^{2}+0.3397 P\right]$

where $P=\left(F_{\mathrm{o}}^{2}+2 F_{\mathrm{c}}^{2}\right) / 3$

$(\Delta / \sigma)_{\max }<0.001$

$\Delta \rho_{\max }=0.41 \mathrm{e} \AA^{-3}$

$\Delta \rho_{\min }=-0.24$ e $\AA^{-3}$

Special details

Geometry. All e.s.d.'s (except the e.s.d. in the dihedral angle between two 1.s. planes) are estimated using the full covariance matrix. The cell e.s.d.'s are taken into account individually in the estimation of e.s.d.'s in distances, angles and torsion angles; correlations between e.s.d.'s in cell parameters are only used when they are defined by crystal symmetry. An approximate (isotropic) treatment of cell e.s.d.'s is used for estimating e.s.d.'s involving 1.s. planes. 
Refinement. Refinement of $F^{2}$ against ALL reflections. The weighted $R$-factor $w R$ and goodness of fit $S$ are based on $F^{2}$, conventional $R$-factors $R$ are based on $F$, with $F$ set to zero for negative $F^{2}$. The threshold expression of $F^{2}>\sigma\left(F^{2}\right)$ is used only for calculating $R$-factors(gt) etc. and is not relevant to the choice of reflections for refinement. $R$-factors based on $F^{2}$ are statistically about twice as large as those based on $F$, and $R$ - factors based on ALL data will be even larger.

Fractional atomic coordinates and isotropic or equivalent isotropic displacement parameters $\left(\hat{A}^{2}\right)$

\begin{tabular}{llllll}
\hline & $x$ & $y$ & $z$ & $U_{\text {iso }} * / U_{\text {eq }}$ & Occ. $(<1)$ \\
\hline O2 & $0.07576(10)$ & 0.0000 & $0.71087(12)$ & $0.0213(2)$ & \\
O6 & $0.37126(7)$ & $0.19208(5)$ & $0.35604(9)$ & $0.02282(19)$ & \\
O7 & $0.28600(10)$ & 0.0000 & $0.13202(12)$ & $0.0194(2)$ & \\
O8 & $0.50741(9)$ & 0.0000 & $0.28277(13)$ & $0.0178(2)$ & \\
O1W & 0.0000 & $0.15469(8)$ & 1.0000 & $0.0266(2)$ & \\
O2W & $0.15495(8)$ & $0.33927(6)$ & $0.11287(12)$ & $0.0282(2)$ & \\
N1 & $0.21286(7)$ & $0.09635(6)$ & $0.51944(9)$ & $0.01624(19)$ & \\
C2 & $0.16226(12)$ & 0.0000 & $0.59004(16)$ & $0.0154(2)$ & \\
C5 & $0.36230(12)$ & 0.0000 & $0.29895(16)$ & $0.0151(2)$ & \\
C6 & $0.31848(9)$ & $0.10501(7)$ & $0.39599(11)$ & $0.0157(2)$ & \\
H1 & $0.1840(14)$ & $0.1584(11)$ & $0.574(2)$ & $0.032(3)^{*}$ & \\
H7 & $0.303(3)$ & $0.058(2)$ & $0.076(5)$ & $0.040(8)^{*}$ & 0.50 \\
H8 & $0.535(3)$ & $-0.056(2)$ & $0.244(4)$ & $0.034(7)^{*}$ & 0.50 \\
H9 & $0.0322(19)$ & $0.1122(16)$ & $0.915(2)$ & $0.055(5)^{*}$ & \\
H10 & $0.1164(17)$ & $0.2799(16)$ & $0.078(2)$ & $0.051(4)^{*}$ & \\
H11A & $0.181(3)$ & $0.373(3)$ & $0.031(5)$ & $0.036(8)^{*}$ & 0.50 \\
H11B & $0.090(3)$ & $0.373(3)$ & $0.160(4)$ & $0.027(6)^{*}$ & 0.50 \\
& & & &
\end{tabular}

Atomic displacement parameters $\left(\AA^{2}\right)$

\begin{tabular}{lllllll}
\hline & $U^{11}$ & $U^{22}$ & $U^{33}$ & $U^{12}$ & $U^{13}$ & $U^{23}$ \\
\hline O2 & $0.0226(4)$ & $0.0201(5)$ & $0.0216(5)$ & 0.000 & $0.0089(3)$ & 0.000 \\
O6 & $0.0270(4)$ & $0.0156(3)$ & $0.0263(4)$ & $-0.0019(2)$ & $0.0091(3)$ & $0.0007(2)$ \\
O7 & $0.0256(5)$ & $0.0181(5)$ & $0.0145(4)$ & 0.000 & $-0.0013(3)$ & 0.000 \\
O8 & $0.0157(4)$ & $0.0151(4)$ & $0.0229(4)$ & 0.000 & $0.0058(3)$ & 0.000 \\
O1W & $0.0331(5)$ & $0.0177(5)$ & $0.0296(5)$ & 0.000 & $0.0112(4)$ & 0.000 \\
O2W & $0.0274(4)$ & $0.0198(4)$ & $0.0369(4)$ & $0.0010(3)$ & $-0.0067(3)$ & $-0.0012(3)$ \\
N1 & $0.0183(3)$ & $0.0130(4)$ & $0.0177(4)$ & $0.0003(2)$ & $0.0041(3)$ & $-0.0011(3)$ \\
C2 & $0.0152(5)$ & $0.0158(5)$ & $0.0153(5)$ & 0.000 & $0.0008(4)$ & 0.000 \\
C5 & $0.0162(5)$ & $0.0153(5)$ & $0.0141(5)$ & 0.000 & $0.0026(4)$ & 0.000 \\
C6 & $0.0171(4)$ & $0.0153(4)$ & $0.0146(4)$ & $0.0001(3)$ & $0.0005(3)$ & $0.0006(3)$ \\
\hline
\end{tabular}

Geometric parameters $\left(\AA,{ }^{\circ}\right)$

\begin{tabular}{llll}
\hline $\mathrm{O} 2-\mathrm{C} 2$ & $1.2183(15)$ & $\mathrm{O} 2 \mathrm{~W}-\mathrm{H} 11 \mathrm{~A}$ & $0.77(4)$ \\
$\mathrm{O} 6-\mathrm{C} 6$ & $1.2132(11)$ & $\mathrm{O} 2 \mathrm{~W}-\mathrm{H} 11 \mathrm{~B}$ & $0.82(3)$ \\
$\mathrm{O} 7-\mathrm{C} 5$ & $1.4000(14)$ & $\mathrm{N} 1-\mathrm{C} 6$ & $1.3666(11)$ \\
$\mathrm{O} 7-\mathrm{H} 7$ & $0.83(3)$ & $\mathrm{N} 1-\mathrm{C} 2$ & $1.3752(9)$ \\
$\mathrm{O} 8-\mathrm{C} 5$ & $1.3810(14)$ & $\mathrm{N} 1-\mathrm{H} 1$ & $0.901(14)$ \\
$\mathrm{O} 8-\mathrm{H} 8$ & $0.78(3)$ & $\mathrm{C} 2-\mathrm{N} 11^{\mathrm{i}}$ & $1.3752(9)$
\end{tabular}




\begin{tabular}{|c|c|c|c|c|c|}
\hline $\mathrm{O} 1 \mathrm{~W}-\mathrm{H} 9$ & \multicolumn{2}{|l|}{$0.868(18)$} & $\mathrm{C} 5-\mathrm{C} 6$ & \multicolumn{2}{|c|}{$1.5272(10)$} \\
\hline $\mathrm{O} 2 \mathrm{~W}-\mathrm{H} 10$ & \multicolumn{2}{|l|}{$0.85(2)$} & $\mathrm{C} 5-\mathrm{C} 6^{\mathrm{i}}$ & \multicolumn{2}{|c|}{$1.5272(10)$} \\
\hline $\mathrm{C} 5-\mathrm{O} 7-\mathrm{H} 7$ & \multicolumn{2}{|l|}{$109(2)$} & \multicolumn{2}{|l|}{$\mathrm{N} 1-\mathrm{C} 2-\mathrm{N} 1^{\mathrm{i}}$} & $117.62(10)$ \\
\hline $\mathrm{C} 5-\mathrm{O} 8-\mathrm{H} 8$ & \multicolumn{2}{|l|}{$112(2)$} & \multicolumn{2}{|l|}{$\mathrm{O} 8-\mathrm{C} 5-\mathrm{O} 7$} & $114.66(10)$ \\
\hline $\mathrm{H} 10-\mathrm{O} 2 \mathrm{~W}-\mathrm{H} 11 \mathrm{~A}$ & \multicolumn{2}{|l|}{$111(3)$} & \multicolumn{2}{|l|}{$\mathrm{O} 8-\mathrm{C} 5-\mathrm{C} 6$} & $108.78(6)$ \\
\hline $\mathrm{H} 10-\mathrm{O} 2 \mathrm{~W}-\mathrm{H} 11 \mathrm{~B}$ & \multicolumn{2}{|l|}{$104(2)$} & \multicolumn{2}{|l|}{$\mathrm{O} 7-\mathrm{C} 5-\mathrm{C} 6$} & $105.27(6)$ \\
\hline $\mathrm{H} 11 \mathrm{~A}-\mathrm{O} 2 \mathrm{~W}-\mathrm{H} 11 \mathrm{~B}$ & \multicolumn{2}{|l|}{$108(3)$} & $\mathrm{O} 8-\mathrm{C} 5-\mathrm{C}^{\mathrm{i}}$ & & $108.78(6)$ \\
\hline $\mathrm{C} 6-\mathrm{N} 1-\mathrm{C} 2$ & \multicolumn{2}{|l|}{$125.49(8)$} & $\mathrm{O} 7-\mathrm{C} 5-\mathrm{C}^{\mathrm{i}}$ & & $105.27(6)$ \\
\hline $\mathrm{C} 6-\mathrm{N} 1-\mathrm{H} 1$ & \multicolumn{2}{|l|}{$117.5(8)$} & $\mathrm{C} 6-\mathrm{C} 5-\mathrm{C} 6^{\mathrm{i}}$ & & $114.19(9)$ \\
\hline $\mathrm{C} 2-\mathrm{N} 1-\mathrm{H} 1$ & \multicolumn{2}{|l|}{$116.2(9)$} & $\mathrm{O} 6-\mathrm{C} 6-\mathrm{N} 1$ & & $122.59(8)$ \\
\hline $\mathrm{O} 2-\mathrm{C} 2-\mathrm{N} 1$ & \multicolumn{2}{|l|}{$121.19(5)$} & $\mathrm{O} 6-\mathrm{C} 6-\mathrm{C} 5$ & & $120.40(7)$ \\
\hline $\mathrm{O} 2-\mathrm{C} 2-\mathrm{N} 1^{\mathrm{i}}$ & $121.19(5)$ & & $\mathrm{N} 1-\mathrm{C} 6-\mathrm{C} 5$ & & $90(7)$ \\
\hline $\mathrm{C} 6-\mathrm{N} 1-\mathrm{C} 2-\mathrm{O} 2$ & $173.91(10)$ & & $\mathrm{O} 7-\mathrm{C} 5-\mathrm{C} 6-\mathrm{O} 6$ & & $59(10)$ \\
\hline $\mathrm{C} 6-\mathrm{N} 1-\mathrm{C} 2-\mathrm{N} 1^{\mathrm{i}}$ & $-6.06(16)$ & & $\mathrm{C} 6-\mathrm{C} 5-\mathrm{C} 6-\mathrm{O} 6$ & & $45(6)$ \\
\hline $\mathrm{C} 2-\mathrm{N} 1-\mathrm{C} 6-\mathrm{O} 6$ & $-170.32(9)$ & & $\mathrm{O} 8-\mathrm{C} 5-\mathrm{C} 6-\mathrm{N} 1$ & & $.99(8)$ \\
\hline $\mathrm{C} 2-\mathrm{N} 1-\mathrm{C} 6-\mathrm{C} 5$ & $13.52(13)$ & & $\mathrm{O} 7-\mathrm{C} 5-\mathrm{C} 6-\mathrm{N} 1$ & & $6(9)$ \\
\hline $\mathrm{O} 8-\mathrm{C} 5-\mathrm{C} 6-\mathrm{O} 6$ & $42.76(12)$ & & $\mathrm{C} 6-\mathrm{C} 5-\mathrm{C} 6-\mathrm{N} 1$ & & $30(13)$ \\
\hline Symmetry code: (i) $x,-y, z$. & & & & & \\
\hline Hydrogen-bond geomet & & & & & \\
\hline$D-\mathrm{H} \cdots A$ & & $D-\mathrm{H}$ & $\mathrm{H} \cdots A$ & $D \cdots A$ & $D-\mathrm{H} \cdots A$ \\
\hline $\mathrm{N} 1-\mathrm{H} 1 \cdots \mathrm{O} 6^{\mathrm{ii}}$ & & $0.901(14)$ & $1.970(14)$ & $2.8580(10)$ & $168.1(13)$ \\
\hline $\mathrm{O} 7-\mathrm{H} 7 \cdots \mathrm{O} 2 W^{\mathrm{iii}}$ & & $0.83(3)$ & $1.92(3)$ & $2.7217(11)$ & $163(3)$ \\
\hline $\mathrm{O} 8-\mathrm{H} 8 \cdots \mathrm{O} 2 W^{\mathrm{iv}}$ & & $0.78(3)$ & $1.98(3)$ & $2.7250(10)$ & $160(3)$ \\
\hline $\mathrm{O} 1 W-\mathrm{H} 9 \cdots \mathrm{O} 2$ & & $0.868(18)$ & 2.075 (19) & $2.9343(9)$ & $170.6(16)$ \\
\hline $\mathrm{O} 2 W-\mathrm{H} 10 \cdots \mathrm{O} 1 W^{\mathrm{v}}$ & & $0.85(2)$ & $1.96(2)$ & $2.8009(11)$ & $171.3(15)$ \\
\hline $\mathrm{O} 2 W-\mathrm{H} 11 A \cdots \mathrm{O} 7^{\mathrm{iii}}$ & & $0.77(4)$ & $1.99(4)$ & $2.7217(11)$ & $160(3)$ \\
\hline $\mathrm{O} 2 W-\mathrm{H} 11 B \cdots \mathrm{O} 8^{\mathrm{vi}}$ & & $0.82(3)$ & $1.96(3)$ & $2.7250(10)$ & $154(3)$ \\
\hline
\end{tabular}

Symmetry codes: (ii) $-x+1 / 2,-y+1 / 2,-z+1$; (iii) $-x+1 / 2,-y+1 / 2,-z$; (iv) $x+1 / 2, y-1 / 2, z$; (v) $x, y, z-1$; (vi) $x-1 / 2, y+1 / 2, z$. 